\title{
Prognostic indicators for visual outcomes following surgery for epiretinal membrane associated with diabetic patients
}

\begin{abstract}
Purpose: To evaluate the predicting factors for good VA after PPV for ERM associated with DME.

Methods: The medical records 29 eyes seen in the Retina Clinic and diagnosed to have ERM associated with DME were reviewed. Investigating potential predicting factors for achieving good vision included a mean change in best-corrected visual acuity (BCVA) before and after PPV, means change in CMT before and after PPV, focal and broad attachment of ERM to the ILM, age, sex and duration of the disease.

Results: In the current study, 29 eyes (19 $(65.5 \%$ OD and 10 (34.5\% OS) of 29 patients were recruited. The mean (SD) age of our sample was 60.8 (10.6) years, range [33-78]. Comparing the mean (SD) LogMAR of preoperative and the last follow-up values, the detected difference was found to be statistically significant $(0.5(0.3), .95 \%$ CI: $0.394-$ 0.608]; $\mathrm{p}<0.0001)$. The mean (SD) OCT decreased postoperatively to 352 (128) micron, then to 313 (101.5) micron in the last follow-up visit assessment, where such decrease from preoperative to the last follow-up assessment was statistically significant (139.2 (92.5) micron, [95\% CI: 103.311-175.046]; $p<0.0001$ ). Investigating potential predicting factors for achieving vision $\geq 20 / 60$, patients who entered the cohort in a relatively higher age and with a better initial visual acuity were found to be more likely to achieve better postoperative vision ( $p=0.016$ and 0.004 for age and visual acuity respectively).
\end{abstract}

Conclusion: Pars plana Vitrectomy for ERM peeling associated with DME showed a better visual outcome. Patients with good initial VA achieved a better final visual outcome. Postoperative improvement in vision is slow. OCT appearance cannot predict visual outcome.

Keywords: diabetic patients, ERM, ILM, epiretinal membrane, OCT, BCVA
Volume 7 Issue 7 - 2017

\author{
Ali M Al-Halafi \\ Department of Ophthalmology, Security Forces Hospital \\ Program, Saudi Arabia
}

\begin{abstract}
Correspondence: Ali M Al-Halafi, Consultant and Vitreoretinal Surgeon, Department of Ophthalmology, Security forces Hospital Program (SFHP), PO Box 3643, Riyadh I I48I, Saudi Arabia,Tel +966-I-8024444, Email amm-ry@hotmail.com
\end{abstract}

Received: November 21, 2017 | Published: December 12, 2017

\section{Introduction}

The epiretinal membrane (ERM) is semitransparent; fibrocellular membrane found on the inner surface of the internal limiting membrane (ILM) at the macula. ${ }^{1}$ ERM most commonly take place in the elderly population and can lead to distortion, blurring of vision, and in some patients severe vision loss. ${ }^{1-3}$ ERM formation may lead to visual inconveniences, such as metamorphopsia, photopsia, blurred vision, and decreased visual acuity, which impacts an individual's quality of life. Machemer $\mathrm{R},{ }^{1,4}$ was the first to describe pars plana vitrectomy (PPV) with ERM peeling procedures. ERMs are documented to regress in around a quarter of cases. ${ }^{5}$ The invention of optical coherence tomography (OCT) has demonstrated a more precise visualization of the macular anatomy and better understanding of the pathophysiology of the vitreoretinal interface diseases. ${ }^{6-8}$ OCT has developed to become the main standard for confirming, managing and follow-up of different vitreoretinal pathology including ERM since described by Puliafito et al. ${ }^{9}$

\section{Patients and methods}

The records of 29 patients seen in the Retina Clinic in our hospital, Riyadh, Saudi Arabia, from January 2011 to April 2015 were retrospectively reviewed. The study was approved by the research committee (RN: 16-192-26). Charts were examined for demographic data (age and gender), initial and final best-corrected Snellen visual acuities, results of slit-lamp examination of the anterior segment, results of dilated fundus examination, and results of OCT. Diabetic macular edema patients with an epiretinal membrane were identified. Other causes of ERM were excluded such as trauma, uveitis, prior retinal detachment and vascular occlusive diseases. All OCTs of both eyes were reviewed, establishing the presence of an epiretinal membrane, which was defined as a thin, smooth hyper-reflective layer between the neural retina and the vitreous (Figure 1). SD-OCT images of all involved eyes at their preoperative examination and postoperative visits at 1 month, 3 months and 6 months were examined. Focal or broad attachment of ERM to the internal limiting membrane (ILM) of the retina, central macular thickness, and inner segment/outer segment (IS/OS) junction was evaluated. LogMAR transformation was used to determine mean acuities and converted back to Snellen acuities for reporting. ERM with a decrease in visual acuity and progression of clinical symptoms were treated with surgical intervention. All patients had standard three-port pars plana vitrectomy (PPV). ERM removal was done with suitable forceps with the help of a staining dye if it is necessary intra-operatively. Our goal was to evaluate the potential predicting factors for achieving good vision. We included a mean change in best-corrected visual acuity (BCVA) before and after PPV for ERM removal, age, sex, duration of the disease and OCT findings such as the attachment of ERM to ILM of the retina, central macular thickness, and integrity of (IS/OS) junction. 


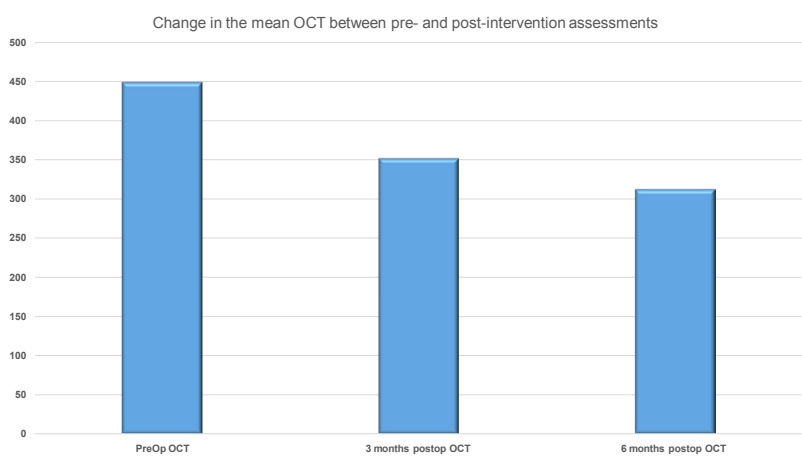

Figure I Preoperative and postoperative OCT Results.

\section{Statistical analysis}

Data were collected and stored in a spreadsheet using Microsoft Excel $2010 \AA$ software. Data management and coding were both done in Excel. Data were analyzed using SPSS ${ }^{\circledR}$ version 20.0 (IBM Inc., Chicago, Illinois, USA). Descriptive analysis was done where categorical variables were presented as frequencies and percentages and continuous variables as the mean and standard deviation $( \pm \mathrm{SD})$. Inferential analysis was done where Chi-test (Fisher exact test whenever indicated) was used to detect the association between potential predicting factors and achieving post-intervention LogMAR VA of $\geq 20 / 60$. Wilcox on Signed rank test was used to investigate any significant difference between baseline, postoperative and last followup visit assessment of LogMAR mean VA and OCT values. Confidence interval level was set to $95 \%$ where a corresponding $p$-value threshold was identified as 0.05 . Accordingly, values $<0.05$ were interpreted as denoting statistical significance.

\section{Results}

In the current study, 29 eyes (19 (65.5\% OD and $10(34.5 \%$ OS) of 29 patients were recruited. The mean (SD) age of our patients was 60.8 (10.6) year, range [33-78] year. Male patients were almost two folds of the females (19 (65.5\%) male to $10(34.5 \%)$. Regarding diagnosis, the majority of eyes were having ERM $(26 ; 89.6 \%)$ ERM with DME and 3 eyes with ERM without DME (3; 10.3\%). In all 29 eyes with ERM confirmed clinically; ERM could be demonstrated with OCT. Central macular thickness was measured. The mean (SD) OCT decreased postoperatively to 352 (128) micron. Then to 313 (101.5) micron after 6 months of follow-up visit assessment, where such decrease from preoperative to the last follow-up assessment was statistically significant (139.2 (92.5), [95\% CI: 103.311-175.046]; $p<0.0001)$ (Figure 1). The inner retinal layers were evaluated preoperatively. The focal attachment of ERM was found in $9(31 \%)$ patients. Broad attachments of ERM was found in $20(69 \%)$ patients which cause restraining of the inner retinal layer and wrinkling and irregularities on the inner retinal surface (Figure 2). There was no difference in visual acuity results after 6 months postoperatively between two entities. Changing of foveal contour with macular thickening in 26 (90\%) eyes was shown preoperatively. Three eyes had a breakdown of photoreceptor IS-OS line which associated with poor vision after 6 months.

Assessment of clinical indices at presentation showed that the mean (SD) LogMAR visual acuity was $1.1(0.3)$. The most prevalent Snellen VA was 20/400 (9; 31\%), followed by 20/200 (7; 24.1\%) and 20/100 (4; 13.8\%), while, the mean (SD) OCT was 449.1 (112.5). In the postoperative assessment, the mean (SD) LogMAR has slightly increased from a preoperative value of $1.1(0.3)$ to $1.6(0.6)$ in the first follow-up then gradually improved to $1.1(0.6)$ in the second followup assessment till it reached 0.6 (0.3) after 6 months of follow-up visit assessment. Comparing preoperative to the last follow-up values, the detected difference was found to be statistically significant $(0.5$ (0.3), [95\% CI: $0.394-0.608] ; \mathrm{p}<0.0001$ ) (Figure 3). Comparison of the ratio of those with better and worse visions showed that $14 / 29$ (48.3\%) entered the cohort with a vision $>=20 / 200$ while the outcome of surgical removal of ERM showed that 25/29 (86.2\%) left the cohort with a vision $>=20 / 200 ; p=0.076$ (Table 1$)$.

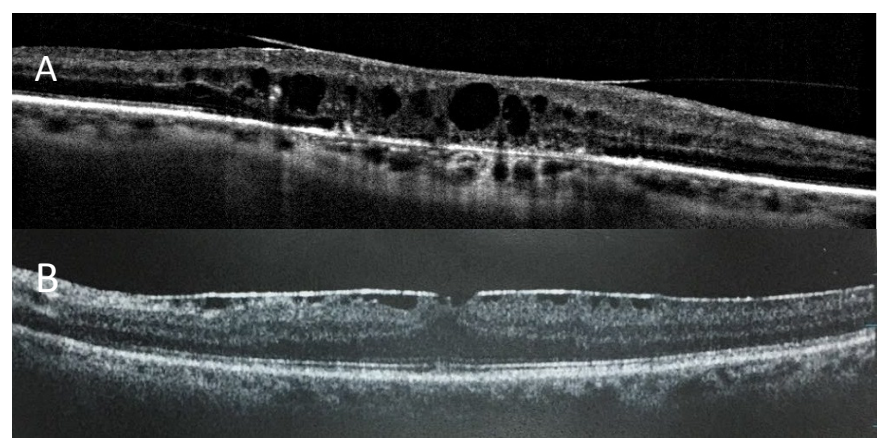

Figure 2 Broad attachment of $\operatorname{ERM}(A)$. Focal attachment of ERM (B).

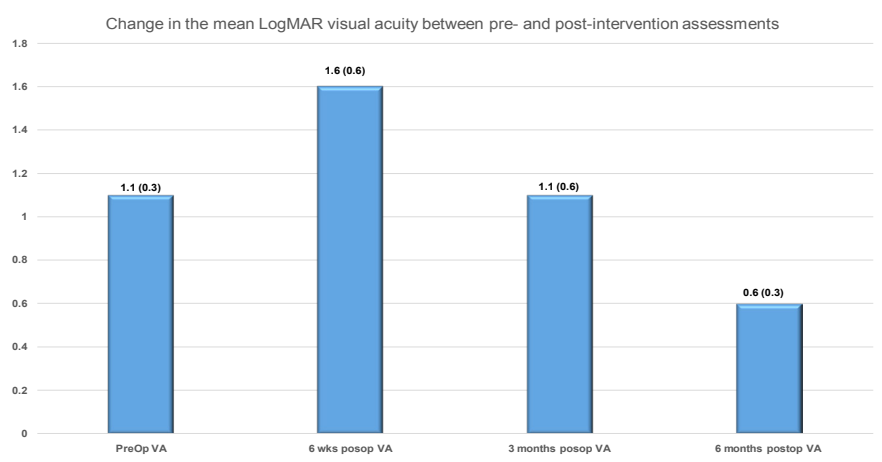

Figure 3 Preoperative and postoperative visual acuity Results.

Table I Visual acuity change chart

\begin{tabular}{lllll}
\hline & & \multicolumn{2}{l}{$\begin{array}{l}\text { Pre-intervention Visual } \\
\text { acuity }\end{array}$} & Total \\
& & $<=20 / 200$ & $>=20 / 200$ & \\
\hline \multirow{2}{*}{ Post-Intervention } & $20 / 50-20 / 160$ & 8 & 4 & 7 \\
& $<=20 / 40$ & 3 & 10 & 18 \\
Total & $<200$ & 4 & 0 & 4 \\
& & 15 & 14 & 29
\end{tabular}

Investigating potential predicting factors for achieving vision $\geq 20 / 60$, patients who entered the cohort in a relatively higher age and with a better initial visual acuity were found to be more likely to achieve better postoperative vision ( $p=0.016$ and 0.004 for age and visual acuity respectively). In patients with ERM, focal or broad attachments preoperatively gave the same visual outcomes after 6 months. The intact photo receptor layer preoperatively has found to be of great significance prognostic factor of postsurgical visual outcomes. BCVA and SD-OCT were analyzed pre and post-operatively. The duration to achieve BCVA after surgery was6 months (Table 2). 
Table 2 Factors predicting good vision $>=20 / 60$

\begin{tabular}{lllll}
\hline Variable & & $\begin{array}{l}\text { Improved } \\
\text { to }>=20 / 60\end{array}$ & $\begin{array}{l}\text { Improved } \\
\text { to }<20 / 60\end{array}$ & P value \\
\hline Age & Mean (SD) & $67.4(7.3)$ & $57.9(10.6)$ & 0.016 \\
VA & Mean (SD) & $0.86(0.22)$ & $1.2(0.32)$ & 0.004 \\
OCT & Mean (SD) & $440.9(90.9)$ & $452.9(122.9)$ & 0.799 \\
Duration (day) & Mean (SD) & $16.8(22.7)$ & $14.9(16.9)$ & 0.573 \\
Sex & & No. $(\%)$ & No. $(\%)$ & \\
& Male & $7(36.8)$ & $12(63.2)$ & 0.351 \\
& Female & $2(20)$ & $8(80)$ & \\
Eye & & & & \\
& & $5(26.3)$ & $14(73.7)$ & 0.449 \\
& OD & $4(40)$ & $6(60)$ & \\
\hline
\end{tabular}

\section{Discussion}

The prevalence of epiretinal membrane (ERM) was about $27 \%$ in eyes with DME. ${ }^{10}$ Few authors reported improvement in bestcorrected visual acuity (BCVA) and level of the diabetic macular edema following PPV and removal of the ERM. ${ }^{11,12}$ The central macular thickness was measured. The mean (SD) OCT decreased postoperatively after6 months of follow-up visit assessment, where such decrease from preoperative to the last follow-up assessment was statistically significant $(p<0.0001)$. The pattern of ERM attachment to ILM was studied. In $31 \%$ of eyes with ERM in our cohort, a focal pattern of attachment to ILM of retina $t$ was documented. The focal pattern showed a more folds into ridge contour which lead to corrugation of the underlying retina. The broad attachment, in contrast, showed less retinal surface undulating and less visual distortions. These findings are similar to previous reports about idiopathic and uveitic ERM. ${ }^{13,14}$ Mori and co-authors also found that around half of the cases with nonidiopathic ERM also showed a focal attachment pattern to the retina. ${ }^{15}$ The present study indicates that there was no difference in visual acuity between either focal or broad attachments of ERM after 6 months of surgery. Up to my knowledge, no commercial SDOCT manufacturer that can generate ERM thickness maps. Even if such automated thickness maps were created, they would be prone to error and artifact. ${ }^{14,16}$ In the current study, ERM thickness was not evaluated. In the present study, the breakdown of IS/OS junction line of the photoreceptors layer was found in 3 eyes preoperatively which showed no improvement in visual outcome after PPV for ERM removal. Previous studies showed the same results. ${ }^{14,17}$

The results of the current study showed gradual improvement in visual outcome following PPV for ERM peeling. Comparing Preoperative to 6 months postoperative follow-up visit assessment, the detected difference was found to be statistically significant $(p<0.0001)$. The biggest gain of vision was shown in those eyes with the better initial visual acuity before surgery. Similarly, with other conditions of the macula in which interventions become ineffective once the VA deteriorates severely. ${ }^{1,18-21}$ The link between anatomical and functional recovery after ERM removal is controversial. ${ }^{22-24}$ In this study, significant improvement in visual acuity and macular thickness was observed after6 months of surgery. Anatomical recovery Precedes improvement in visual acuity. ${ }^{25}$ Also, improvement in visual acuity has been reported years after surgery. ${ }^{26}$ According to previous studies, persistence metamorphopsia, and contrast sensitivity, despite the anatomical recovery, may affect the evaluation of subjective visual recovery. ${ }^{27,28}$ However, in the present study, we did not investigate subjective functional improvement, such as metamorphopsia and contrast sensitivity other than visual acuity.

Investigating potential predicting factors for achieving vision $\geq 20 / 60$, patients who entered the cohort in a relatively higher age and with a better initial visual acuity were found to be more likely to achieve better postoperative vision ( $p=0.016$ and 0.004 for age and visual acuity respectively). In patients with ERM, focal or broad attachments preoperatively gave the same visual outcomes after 6 months. Different studies have shown a direct correlation between the integrity of the photoreceptor inner segment/outer segment (IS/ OS) junction line preoperatively and have proven to be an important indicator of postoperative visual acuity. ${ }^{29}$ In the study of Kitaya et al.. ${ }^{28}$ Postoperative vision $\geq 0.7$ was correlated with good integrity of the IS/OS junction. However, Sano et al., ${ }^{30}$ found that intact IS/ OS line was not a reliable prognostic factor in the early postoperative period with the achievement of a repaired IS/OS line at 6 months.

Eyes with none intact IS/OS junction had significantly lower postoperative best-corrected visual acuity (BCVA) ${ }^{27,31}$ Similarly, Falkner-Radler et al., ${ }^{17}$ reached that intact IS/OS junction was an important factor to expect the visual outcomes after ERM peeling confirmed by TD-OCT and SD OCT. In addition, Inoue et al. ${ }^{6}$ showed that the intact IS/OS cohort had better final visual acuity than none intact IS/OS cohort. The use of a dye for ERM peeling is unlikely to be a significant factor in the final visual acuity results. A brilliant blue-assisted internal limiting membrane (ILM) peeling was in every surgery. Similarly, another study also did not show a significant correlation between preoperative central foveal thickness, the presence of a macular pseudo hole or retinal cysts with the postoperative visual acuity. ${ }^{16,27}$ Our numbers of small-gauge PPV surgeries were considered as small to permit evaluation of that factor. However, other studies have shown faster visual gain and fewer complications with smallgauge trans-conjunctival suture less vitrectomy procedures for ERM peeling. ${ }^{1,32,33}$ To conclude, PPV for ERM peeling associated with DME showed a better visual outcome. Patients with good initial VA achieved a better final visual outcome. Postoperative improvement in vision is slow. OCT appearance cannot predict visual outcome. ${ }^{34}$

\section{Acknowledgments}

None.

\section{Conflicts of interest}

Author declares that there are no conflicts of interest.

\section{Funding}

None.

\section{References}

1. Dawson SR, Shunmugam M, Williamson TH. Visual acuity outcomes following surgery for idiopathic epiretinal membrane: an analysis of data from 2001 to 2011. Eye (Lond). 2014;28(2):219-224.

2. Jacobsen CH. Epiretinal membranes. Optom Clin. 1996;5(1):77-94. 
3. Wong JG, Sachdev N, Beaumont PE, et al. Visual outcomes following vitrectomy and peeling of epiretinal membrane. Clin Exp Ophthalmol. 2005;33(4):373-378.

4. Machemer R. The surgical removal of epiretinal macular membranes (macular puckers) (author's transl). Klin Monbl Augenheilkd. 1978;173(1):36-42

5. Fraser-Bell S, Guzowski M, Rochtchina E, et al. Five-year cumulative incidence and progression of epiretinal membranes: the Blue Mountains Eye Study. Ophthalmology. 2013;110(1):34-40.

6. Arevalo JF, Lasave AF, Arias JD, et al. Clinical applications of optical coherence tomography in the posterior pole: the 2011 Jose Manuel Espino Lecture: part II. Clin Ophthalmol. 2013;7:2165-2179.

7. Arevalo JF, Lasave AF, Arias JD, et al. Clinical applications of optical coherence tomography in the posterior pole: the 2011 Jose Manuel Espino Lecture: part ' II." Clin Ophthalmol. 2013;7:2181-2206.

8. Adhi M, Duker JS. Optical coherencetomography current and future applications. Current Curr Opin Ophthalmol. 2013;24(3):213-221.

9. Puliafito CA, Hee MR, Lin CP, et al. Imaging of macular diseases with optical coherence tomography. Ophthalmol. 1995;102(2):217-229.

10. Yamamoto T, Akabane N, Takeuchi S. Vitrectomy for diabetic macular edema: the role of posterior vitreous detachment and epimacular membrane. Am J Ophthalmol. 2001;132(3):369-377.

11. Gandorfer A, Rohleder M, Grosselfinger S, et al. Epiretinal pathology of diffuse diabetic macular edema associated with vitreomacular traction. $\mathrm{Am}$ J Ophthalmol. 2005;139(4):638-652.

12. Sakimoto S, Saito Y, Nakata K, et al. Surgical outcomes of epiretinal membrane removal after successful pars plana vitrectomy for retinal diseases. Jpn J Ophthalmol. 2008;52(3):227-230.

13. Kim JS, Chhablani J, Chan CK, et al. Retinal adherence and fibrillary surface changes correlate with surgical difficulty of epiretinal membrane removal. Am J Ophthalmol. 2012;153:692-697.

14. Nazari H, Dustin L, Heussen FM, et al. Morphometric spectraldomain optical coherence tomography features of epiretinal membrane correlate withvisual acuity in patients with uveitis. Am J Ophthalmol. 2012;154(1):78-86.

15. Chang S, Gregory-Roberts EM, Park S, et al. Double peeling during vitrectomy for macular pucker. JAMA Ophthalmol. 2013;131:525-530.

16. Inoue $\mathrm{M}$, Morita $\mathrm{S}$, Watanabe $\mathrm{Y}$, et al. Inner segment/outer segment junction assessed by spectral-domain optical coherence tomography in patients with idiopathic epiretinal membrane. Am J Ophthalmol. 2010;150:834-839

17. Falkner-Radler CI, Glittenberg C, Hagen S, et al. Spectral-domain optical coherence tomography for monitoring epiretinal membrane surgery. Ophthalmology. 2010;117(4): 798-805.

18. Hiscott PS, Unger WG, Grierson I, et al. The role of inflammation in the development of epiretinal membranes. Curr Eye Res. 1998;7(9):877-892.
19. Flaxel CJ, Edwards AR, Aiello LP, et al. Factors associated with visual acuity outcomes after vitrectomy for diabetic macular edema: diabetic retinopathy clinical research network. Retina. 2010;30(9):1488-1495.

20. Schulze SD, Hesse L. Tissue plasminogen activator plus gas injection in patients with subretinal hemorrhage caused by age-related macular degeneration: predictive variables for visual outcome. Graefes Arch Clin Exp Ophthalmol. 2002;240(9):717-720.

21. Smiddy WE. Clinical applications of cost analysis of diabetic macular edema treatments. Ophthalmology. 2012;119(12):2558-2562.

22. Massin P, Allouch C, Haouchine B, et al. Optical coherence tomography of idiopathic macular epiretinal membranes before and after surgery. $\mathrm{Am}$ J Ophthalmol. 2000;130(6):732-739.

23. Niwa T, Terasaki H, Kondo M, et al. Function and morphology of macula before and after removal of idiopathic epiretinal membrane. Invest Ophthalmol Vis Sci. 2003;44(4):1652-1656.

24. Wilkins JR, Puliafito CA, Hee MR, et al. Characterization of epiretinal membranes using optical coherence tomography. Ophthalmology. 1966;103(12):2142-2151.

25. Grewing R, Mester U. Results of surgery for epiretinal membranes and their recurrences. Br J Ophthalmol. 1996;80(4):323-326.

26. McDonald HR, Verre WP, Aaberg TM. Surgical management of idiopathic epiretinal membranes. Ophthalmology. 1986;93(7):978-983.

27. Margherio RR, Cox MS, Trese MT, et al. Removal of epimacular membranes. Ophthalmology.1985;92(8):1075-1083.

28. Rice TA, De Bustros S, Michels RG, et al. Prognostic factors in vitrectomy for epiretinal membranes of the macula. Ophthalmology. 1986;93(5):602610.

29. Kitaya N, Hikichi T, Kagokawa H, et al. Irregularity of photoreceptor layer after successful macular hole surgery prevents visual acuity improvement. Am J Ophthalmol. 2004;138(2):308-310.

30. Goldberg RA, Waheed NK, Duker JS. Optical coherence tomography in the preoperative and postoperative management of macular hole and epiretinal membrane. Br J Ophthalmol. 2004;98(Suppl 2):ii20-ii23.

31. Sano M, Shimoda Y, Hashimoto H, et al. Restored photoreceptor outer segment and visual recovery after macular hole closure. The American Journal of Ophthalmology. 2009;147(2):313.e1-318.e1.

32. Suh MH, Seo JM, Park KH, et al. Associations between macular findings by optical coherence tomography and visual outcomes after epiretinal membrane removal. Am J Ophthalmol. 2009;147(3):473-480.

33. Valmaggia C. Pars plana vitrectomy with 25-gauge instruments in the treatment of idiopathic epiretinal membranes. Klin Monbl Augenheilkd. 2007;224(4):292-296.

34. Rizzo S, Genovesi-Ebert F, Murri S, et al. 25-gauge, sutureless vitrectomy and standard 20-gauge pars plana vitrectomy in idiopathic epiretinal membrane surgery: a comparative pilot study. Graefes Arch Clin Exp Ophthalmol. 2006;244(4):472-479. 